\title{
Las asesorías pedagógicas universitarias en la Argentina
}

\section{As assessorias pedagógicas universitárias na Argentina}

\section{University educational consultants in Argentina ${ }^{1}$}

\author{
Elisa Lucarelli²
}

\begin{abstract}
RESUMEN
Las circunstancias que se presentan a nivel mundial en estas primeras décadas del siglo XXI obligan a la universidad, una vez más, a repensarse a sí misma, a su proyecto político y a las prácticas que en ella se realizan. En este artículo se presentan algunos resultados de una investigación que lleva a cabo un equipo de la Universidad de Buenos Aires, preocupado por identificar estrategias institucionales que puedan contribuir al mejoramiento de la calidad de la enseñanza universitaria. Particulariza su foco investigativo en el asesor pedagógico universitario en instituciones públicas argentinas. Se interesa por abordar aspectos relativos al rol que cumplen estos actores, en torno a dos ejes fundamentales: la descripción de estas asesorías en su contexto institucional y según su estructura organizativa, y la comprensión de la problemática central alrededor de la cual nuclean sus actividades. En el estudio se sigue una metodología cualitativa, fundamentándose en el análisis de Comunicaciones sobre esta temática, escritas por esos actores y en entrevistas en profundidad. Los núcleos considerados en el presente artículo se centran en las acciones de asesoramiento como forma de acción directa en los problemas del aula y la institución, y en las de investigación como marca de jerarquización académica. Algunos resultados permiten
\end{abstract}

DOI: $10.1590 / 0104-4060.42057$

1 Financiado por la Secretaría de Políticas Universitarias de Argentina, Proyecto PPCP003/11.

2 Universidad de Buenos Aires. Ciudad Autónoma de Buenos Aires, Argentina. Puan, 480. E-mail: elisalucarelli@arnet.com.ar 
afianzar el supuesto que ubica al asesor pedagógico uno de los sujetos que contribuyen a la construcción de la Didáctica y Pedagogía Universitarias.

Palabras-clave: asesor pedagógico; universidad; intervención; investigación.

\title{
RESUMO
}

As circunstâncias que se apresentam em todo o mundo nas primeiras décadas do século XXI forçam a universidade, mais uma vez, a repensar a si própria, seu projeto político e as práticas que são realizadas. Este artigo apresenta alguns resultados de uma investigação realizada por uma equipe da Universidade de Buenos Aires, preocupada em identificar estratégias institucionais que podem contribuir para a melhoria da qualidade do ensino universitário. Centra seu foco investigativo no assessor pedagógico universitário de instituições públicas argentinas e está interessada em questões relativas ao papel desses atores, em torno de dois eixos fundamentais: a descrição destes assessores em seu contexto institucional, de acordo com sua estrutura organizacional, e a compreensão do problema central em torno do qual se reúnem as suas atividades. $\mathrm{O}$ estudo segue uma metodologia qualitativa, com base na análise das Comunicações sobre este assunto, escritas por esses atores e em análise intensa de entrevistas. Os núcleos considerados neste artigo centram-se nas ações de assessoramento como uma forma de ação direta sobre os problemas da sala de aula e da instituição e na pesquisa como um indicador de ranking acadêmico. Alguns resultados permitem reforçar a hipótese de que o assessor pedagógico é um dos sujeitos que contribui para a construção da Didática e Pedagogia Universitárias.

Palavras-chave: assessor pedagógico; universidade; intervenção; pesquisa.

\begin{abstract}
Circumstances that occur worldwide in the first decades of the $21^{\text {st }}$ century force University, once again, to rethink about itself, its political project and the practices that are carried out. This article presents some results of an investigation developed by a team from the University of Buenos Aires, worried to identify institutional strategies that may contribute to the improvement of the quality of university education. It details its investigative focus on the Pedagogical University Advisor in Argentinean public institutions. It has an interest in addressing issues relating to these actors' role, around two fundamental axes: the description of these consultants in their institutional context, according to its organizational structure, and the understanding of the central problem around which their activities are gathered. The study follows a qualitative methodology, based on the analysis of Papers on this subject, written by those actors, and of interviews in depth. The kernels
\end{abstract}


considered in this article are focused on the actions of counseling as a form of direct action on the classrooms and the institution's problems, and on the research as a mark of academic ranking. Some results allow to strengthening the course that the Pedagogical Advisor is one of the subjects that contribute to the construction of University Didactics and Pedagogy.

Keywords: pedagogical advisor; university; intervention; research.

\section{Las asesorías pedagógicas universitarias en la Argentina}

Las circunstancias que se presentan a nivel mundial en estas primeras décadas del siglo XXI obligan a la universidad, una vez más, a repensarse a sí misma, a su proyecto político y a las prácticas que en ella se realizan. El colapso mundial de las economías, en Estados Unidos en los primeros años y en los países menos desarrollados de la Unión Europea en la actualidad, marcan una alerta en cuanto a la vigencia del modelo neoliberal conservador, al mismo tiempo que en la región latinoamericana aparecen condiciones políticas propicias para el desarrollo de un pensamiento y de propuestas de acción más allá de la regulación del mercado. La universidad, acosada por requerimientos de ese entorno para modificar sus prácticas según modelos ajenos a su propia idiosincrasia, parece responder lentamente a la urgencia de plantearse nuevas definiciones.

En América Latina la necesidad de una universidad con pensamiento autónomo y compromiso ético, que se expresa a través de prácticas pertinentes, alejadas de la torre de marfil, se da en tiempos de consolidación de democracias en contexto de pobreza, marcadas por el respeto a la diversidad y con eje en la tolerancia.

Las Ciencias Sociales en los últimos años han hecho foco, a través de sus investigaciones, en estas problemáticas. Dentro de ellas la Pedagogía y la Didácticas universitarias surgen y se afianzan poco a poco como parte de un espacio de reflexión y de acción para dar respuesta a los problemas que la docencia encuentra cotidianamente en el contexto universitario.

Con esa intencionalidad y entendiendo a la investigación científica como práctica social anclada en un determinado contexto sociohistórico, que tiene su génesis en la problematización de la realidad, y cuya finalidad es generar conocimiento científico con el fin de transformar la realidad (SIRVENT, 2006, p. 6-7), desde el Programa Estudios sobre el aula universitaria del Instituto de Investigaciones en Ciencias de la Educación de la Facultad de Filosofía y Letras de la Universidad de Buenos Aires, se están desarrollando investigaciones cen- 
tradas en el análisis de los procesos que se dan en el aula universitaria y de las estrategias que lleva a cabo la institución para favorecer situaciones alternativas a la enseñanza tradicional. Dentro de estas acciones se lleva a cabo la investigación, eje de este artículo, sobre las asesorías pedagógicas universitarias en la Argentina.

Ella forma parte de una acción interinstitucional y binacional Argentina-Brasil e se trata del Proyecto Conjunto de Investigación Mercosur Argentina Secretaría de Políticas Universitarias (SPU) - Brasil, Coordenação de Aperfeiçoamento de Pessoal de Nível Superior (CAPES) Programa de Proyectos Conjuntos de Investigacíon en el Mercosur (PPCP) 003/11, Estrategias institucionales para el mejoramiento de la calidad de la Educación Superior y el desarrollo profesional docente en el que participan equipos de universidades de ambos países y de Uruguay. El Grupo brasileño, con sede en la Universidade do Vale do Rio dos Sinos (UNISINOS) ${ }^{3}$, y el Grupo argentino Estudios en el aula universitaria de la Universidad de Buenos Aires ${ }^{4}$ han desarrollado, a lo largo de más de veinte años de trabajo colaborativo, marcos teóricos y metodológicos en común que les han permitido producciones y presentaciones conjuntas en muchos eventos académicos a lo largo de estos años. En la actualidad ambos equipos encaran esa investigación en común sobre las estrategias para mejorar la calidad de la enseñanza en el nivel, con énfasis en las asesorías pedagógicas, enmarcadas en sus culturas institucionales. Interesa mostrar cómo, a partir del reconocimiento de trayectorias sociales e institucionales diferentes, los dos grupos se preocupan por esas estrategias que afectan o pueden afectar la vida de los académicos en pos de una mayor profesionalización.

Orientado por el objetivo general de analizar las prácticas y las experiencias de mejoramiento de la calidad pedagógica en las universidades de los dos países, identificando estrategias institucionales de intervención y acompañamiento de los procesos pedagógicos, el proyecto concreta intencionalidades y formas de investigación centradas en acciones que implican la participación y la articulación comparativa en un doble circuito. El primero está focalizado en la conformación de un entramado institucional en torno a cada sede (UNISINOS y Universidad de Buenos Aires - UBA), donde a partir de estas coordinaciones cada una de las universidades involucradas en el proyecto va llevando a cabo estudios particulares, siempre vinculados por un marco teórico y metodológico

3 Coordinado por Dr ${ }^{\mathrm{a}}$ María Isabel da Cunha (UNISINOS) e integrados por equipos investigadores de varias universidades brasileñas.

4 Coordinado por Elisa Lucarelli (Universidad de Buenos Aires/Universidad Nacional de Tres de Febrero) y los investigadores Claudia Finkelstein (Universidad de Buenos Aires), Viviana Solberg (Universidad de Buenos Aires), Alicia Villagra (Universidad de Tucumán), Ana Maria Malet (Universidad Nacional del Sur), de Argentina, y Mercedes Collazo, Sylvia De Bellis, Patricia Perera, Vanesa Sanguinetti (Universidad de la República) de Uruguay. 
común. El segundo circuito supone la comparación de avances, metodologías $\mathrm{y}$ resultados de las investigaciones de Brasil por un lado, y de Argentina y Uruguay por el otro. En esta relación dialéctica entre generalidad del trabajo conjunto regional y especificidad de las acciones institucionales asumidas en la investigación, el proyecto liderado por la UNISINOS aborda la temática de las asesorías pedagógicas universitarias como uno de sus núcleos de indagación, a la par que enfatiza la identificación de otras estrategias institucionales. En el caso del proyecto liderado por la UBA, la investigación está centrada exclusivamente en el análisis del rol de ese actor institucional, en su contexto histórico y situacional. (DA CUNHA; LUCARELLI, 2013).

Las asesorías pedagógicas universitarias no es un tema nuevo para el equipo responsable de este Proyecto binacional por Argentina, dado que ha incorporado como parte de sus integrantes, a asesores pedagógicos de distintas Unidades Académicas desde su constitución en la década del 80, a la vez que desarrolló estudios y seminarios de formación alrededor de su problemática. (LUCARELLI, 2000).

En esta línea el equipo UBA se planteó estos objetivos como específicos del presente trabajo investigativo:

- identificar y explorar las formas institucionales de apoyo pedagógico, sus estrategias y los resultados alcanzados;

- analizar trayectorias institucionales relacionadas con el asesoramiento pedagógico y con la posibilidad de implementar innovaciones en las prácticas educativas;

- discutir los saberes involucrados en el asesoramiento pedagógico universitario, en las dimensiones procesuales e investigativas.

En consecución con estos objetivos el foco está puesto en abordar aspectos relativos al rol que cumplen los asesores pedagógicos universitarios, en torno a dos ejes fundamentales: la descripción de las APU en su contexto institucional y según su estructura organizativa, y la comprensión de la problemática central alrededor de la cual nuclean sus actividades.

En este artículo se trabajará con algunos resultados de la investigación específica que lleva a cabo el equipo del Programa de la Universidad de Buenos Aires, partiendo del análisis de ponencias presentadas por los asesores pedagógicos de universidades nacionales en un evento académico sobre el tema y que diera lugar a una obra. (LUCARELLI; FINKELSTEIN, 2012). Se busca conformar un cuadro de situación de núcleos de prácticas desarrolladas por los asesores en distintos campos de actividades.

La investigación desarrolla, en consecuencia, una línea metodológica cualitativa, a partir del análisis transversal de datos provenientes de fuentes documentales ( 20 comunicaciones correspondientes a 24 equipos pedagógicos 
de universidades nacionales argentinas) y de entrevistas en profundidad realizadas a algunos de estos actores, dando lugar así a la generación de categorías sustantivas sobre esta problemática.

\section{Algunas cuestiones contextuales alrededor de este actor en Argentina}

La problemática acerca de la Pedagogía y la Didáctica Universitarias es un tema de larga data en el pensamiento y en las acciones propiciadoras del cambio en las instituciones argentinas. A comienzos del siglo XX, en 1918, la Reforma Universitaria de Córdoba reclamó por principios renovadores a la enseñanza tradicional que se desarrollaba en las aulas. A la vez, el debate y la reflexión acerca de los principios pedagógicos se desarrollaron fundamentalmente a través de la acción de filósofos y políticos, quedando en segundo plano la conformación de un corpus sistemático acerca de qué es enseñar en la universidad. No es sino hasta fines de la década del 50 en que comienza a ser preocupación el desarrollo de recursos genuinamente pedagógicos en la universidad. Es en esta época donde se advierten esfuerzos institucionales organizativos concretados en Unidades de Pedagogía Universitaria y de Orientación Vocacional que brindaban apoyo a estudiantes y profesores universitarios especialmente en las Universidades Nacionales del Litoral (UNL) y Buenos Aires (UBA). Coincide esta época con el surgimiento de un pensamiento latinoamericano en el campo de la ciencia y la tecnología y de la búsqueda por parte de la universidad en transformarse en actor de la planificación del desarrollo nacional. (LANGER, 2008).

La universidad científica, de tradición humboldtiana, se vuelve hacer presente de manera manifiesta en la Argentina oponiéndose al modelo exclusivamente profesionalista. Se valora el profesor como investigador y se apoya el desarrollo de los laboratorios y de los institutos de investigación. Se propicia la modernización de los planes de estudio y la diversificación de las ciencias humanísticas y sociales, dando lugar a la creación de las carreras de Sociología, Psicología, Antropología y a la transformación de la carrera de Pedagogía, de fuerte énfasis filosófico, en la de Ciencias de la Educación, articulada a partir de entonces con el campo social.

En simultáneo las Unidades de Pedagogía Universitaria y de Orientación Vocacional de la década del 60 generan un significativo avance de la presencia de lo pedagógico y didáctico en la universidad argentina, y se concreta a través del surgimiento de grupos de profesionales provenientes de las ciencias sociales, pedagogos, psicólogos, sociólogos, preocupados por los procesos que se dan en 
el aula universitaria. Estos grupos (que desarrollan principalmente actividades de formación de los docentes universitarios en las temáticas de enseñanza, aprendizaje, currículum, las relaciones de la educación con el contexto) derivarán más adelante en los equipos de asesoría pedagógica universitaria. Estos movimientos orientados hacia la renovación de las dinámicas y estructuras de la educación universitaria se ven interrumpidos en épocas dictatoriales, cobrando impulso transformadores con la recuperación de la vida democrática a mediados de la década del 80 .

En la actualidad las asesorías pedagógicas universitarias en Argentina desarrollan sus acciones de formación docente, investigación, intervención y colaboración con la gestión teniendo como foco de atención esa problemática y estableciéndose, en consecuencia, junto a los profesores, como uno de los actores que contribuyen a la conformación del campo de la Didáctica Universitaria.

\section{El asesor pedagógico universitario como sujeto y eje de nuestra investigación}

El reconocimiento de la importancia de estas acciones desde la mirada de los propios actores fue uno de los primeros hallazgos que interesa destacar del estudio que queremos presentar. En efecto el análisis de las Comunicaciones elaboradas por esos equipos pedagógicos permitió ver que ellos reconocen los siguientes campos (ordenados en orden decreciente de frecuencias) como eje de la problemática central de sus acciones: apoyo a proyectos institucionales, formación, asesoramiento, investigación y extensión. (LUCARELLI; FINKELSTEIN; SOLBERG, 2014). De estos campos se seleccionan para desarrollar en este artículo dos: el asesoramiento y la investigación; el asesoramiento por ser esta función la que históricamente fue y es reconocida para dar nombre a los equipos, y la investigación porque se considera que la realización de este tipo de acciones se constituye en un camino que posibilita la valoración de los asesores pedagógicos como actores universitarios asimilables a los docentes y no como meros técnicos de la educación.

\section{El asesoramiento, algo más que un nombre}

Importó ver, ante el análisis de los datos recabados, algunos aspectos conceptuales de manera de poder comprender cuál era el imaginario que en- 
marcaba la mención que hacían los sujetos en estudio cuando señalaban que estaban asesorando.

En este sentido se puede consignar que el asesoramiento refiere, por lo menos, a dos sentidos: a la actividad que alude, da nombre y por tanto marca de manera significativa al rol del asesor pedagógico universitario en la institución, y también al núcleo de tareas específicas relativas a una de las áreas de acción.

Desde ese encuadre referido a su rol, la asesoría pedagógica es reconocida como una profesión de ayuda (LUCARELLI, 2000; LUCARELLI; FINKELSTEIN, 2012) en un medio donde las prácticas de intervención se orientan a lograr cambios que afecten a la institución universitaria como un todo y al aula en particular. De allí que se entienda al asesoramiento enfocando los procesos interactivos de colaboración con las instituciones para prevenir posibles problemas, participar y ayudar en la solución de los que puedan existir y cooperar en la consecución de una mayor mejora educativa. (MURILLO ESTEPA, 1997, p. 44). Considerado como recurso estratégico para el cambio, su accionar implica esclarecimiento de la situación y búsqueda de estrategias necesarias para que la institución pueda elaborar sus propios procesos de cambio, ponerlos en marcha y evaluarlos. (SÁNCHEZ MORENO, 1997, p. 332).

En la conocida clasificación de Havelock (1969) acerca de los roles que asume un agente de cambio para transferir y utilizar conocimientos, el rol del asesor pedagógico universitario se configura como mediador de la información y como aquel facilitador en la identificación y resolución de problemas. La intervención que implican sus acciones hace posible, en un acto reflexivo en común, mirar los problemas desde una nueva mirada.

Uno de los asesores testimonia así...

[...] el docente titular de una asignatura se acerca a la asesoría para plantear un problema ocurrido en su cátedra. Ha descubierto que uno de los auxiliares de su cátedra ha realizado acciones académicas poco éticas. En una primera instancia, desde la asesoría se propuso un trabajo al interior de la cátedra, con reuniones con los docentes y discusiones en relación a cómo resolver este problema. (Universidad Nacional de Entre Ríos - UNER, Facultad de Ingeniería, Área de Asesoría Pedagógica y de Orientación Vocacional).

El asesoramiento se presenta en este encuadre como un campo de interacción (FERNÁNDEZ, 2012) con algunas notas que hacen a la caracterización de una práctica compleja, desde la perspectiva de la articulación de esa interacción así como de los grupos disciplinares profesionales que entran en juego. 
En relación con el primer aspecto conviene destacar a la tarea de asesoramiento como un punto de conjunción de prácticas, ya que se contacta y diferencia de otras prácticas también interactivas como son las propias de la formación, la innovación educativa, la supervisión, la orientación, el apoyo a la investigación y la extensión. El asesoramiento, como ocurre en el desarrollo de cualquiera de ellas, implica poner a disposición de los sujetos involucrados un marco teórico valorativo que permita comprender y justificar el desarrollo de esa práctica en la universidad. El desempeño de un asesor pedagógico en esa institución es revelador de una determinada concepción pedagógica y didáctica, ya que el análisis de las prácticas hace evidente la perspectiva teórica desde donde se definen rumbos y decisiones. Su trabajo cotidiano da cuenta de la existencia de esa concepción, que articula un conjunto de teorías factible de proporcionar orden, claridad y fundamento a las acciones, y que, al mismo tiempo, permite definir qué decisiones se toman y por qué se eligió ese camino de acción y no otro para intervenir en los procesos que se dan en el aula.

El otro aspecto connotativo del asesoramiento es su carácter de tarea cooperativa, que a su vez deriva en dos condiciones: la necesidad de articular campos profesionales disciplinares distintos y la consideración de la disponibilidad del otro para entrar en la tarea de asesoramiento. (FERNÁNDEZ, 2012).

El trabajo cooperativo de asesores pedagógicos y los otros actores de la institución involucrados en el asesoramiento pone de manifiesto el acercamiento de dos campos epistemológicos conformados por procesos históricos asimétricos y con distintas tradición y valoración social: el pedagógico y el disciplinar profesional. Dentro de la tradición académica, influida por los postulados de la ciencia moderna, el contenido específico de las disciplinas asumía (o asume) un valor significativamente mayor que el conocimiento pedagógico (DA CUNHA, 2006), generándose una situación de asimetría, que el asesor pedagógico debe reconocer como punto de partida para la construcción de espacios de trabajo en común con los docentes. Esta construcción puede darse sólo si el pedagogo y el disciplinar pueden reconocer la presencia de actitudes defensivas y resistenciales que impiden la tarea en común. Sólo una mirada que reconozca la especificidad epistemológica y la pertinencia de cada disciplina y las trascienda puede crear las condiciones de disponibilidad necesaria para un encuentro productivo de ambos sujetos, alentando su interés por encontrar vías de resolución apropiadas a los problemas del aula y de la institución en su conjunto. Así expresa un asesor pedagógico:

[...] es frecuente que los docentes de la institución se acerquen a la asesoría a plantear sus preocupaciones respecto de sus asignaturas, 
preocupaciones tales como: la cantidad de alumnos recursantes y/o libres que hubo en determinado año en sus materias; la dificultad que presentan los estudiantes en la comprensión de las consignas de un examen o en la lectura de los textos de la materia; la inasistencia a las clases que aumenta progresivamente con el paso del tiempo de cursado; etc. [...] Lo que suelo hacer ante esto es escucharlos con interés, dando a sus voces un protagonismo principal e intentando comprenderlos en su preocupación. (UNER, Facultad de Ingeniería, Área de Asesoría Pedagógica y de Orientación Vocacional).

El reconocimiento y el respeto por la diversidad y la especificidad que implica cada campo disciplinar profesional se hace presente tanto en la colaboración con los docentes hacia la conformación de modalidades alternativas a las rutinarias para orientar los procesos de enseñanza y de aprendizaje universitarios de manera genuina y creativa, como en el trabajo conjunto con la gestión $\mathrm{y}$ otros sectores para la definición de proyectos que afecten a la institución en su conjunto.

Es muy delicada la acción del Asesor Pedagógico en las mencionadas situaciones ya que: favorece y resguarda las relaciones; hace fluida la circulación de la información, de los aportes disciplinares, metodológicos y organizacionales que hacen los integrantes de los eventuales grupos y sobretodo, capitaliza ese caudal teórico, práctico y empírico, para favorecer las instancias de formación docente del profesor universitario. (UNL, Facultad de Arquitectura, Diseño y Urbanismo).

El asesoramiento requiere además de un trabajo orientado a la generación de un espacio común de reflexión crítica, en el que puede reconocerse un proceso concretado en tres momentos (SANCHEZ MORENO, 1997, p. 334): el inicial, destinado a acordar las condiciones en que se desarrollará el trabajo y donde se exponen expectativas, intenciones y responsabilidades poniendo en juego la disponibilidad de ambos actores para la tarea; el central, de desarrollo de la tarea de intervención; y el de culminación, donde se analiza el trabajo realizado, su impacto y repercusiones. 


\section{La investigación como marca de jerarquización académica}

Es sabido que el fortalecimiento de la investigación científica se puede constituir en un factor significativo para el mejoramiento de la enseñanza y la formación de profesionales. Esto es factible en la medida que se promueva no sólo la participación de estudiantes y docentes en el proceso de creación e invención de nuevos conocimientos, sino que se creen condiciones pedagógicas para la formación en actitudes y habilidades favorables a una concepción constructiva del conocimiento. En esta línea de pensamiento es posible identificar, según lo expresado en las Comunicaciones analizadas, los proyectos de investigación que desarrollan, en el campo de la Pedagogía y la Didáctica Universitarias, más de la mitad de los equipos de asesoría pedagógica indagados.

Cabe considerar que la investigación en este campo manifiesta, en su dinámica y estructura, rasgos propios del campo científico. En este sentido, Langer (2008) retoma la noción de campo de Bourdieu para dar cuenta del funcionamiento que asumen las instituciones de educación superior a través de la creación de criterios relativamente autónomos de las normas sociales, constituyéndose esta perspectiva en una crítica a las ideas mecanicistas sobre el comportamiento de los investigadores. Los miembros de los equipos de investigación, que participan del campo, procuran ampliar su capital científico y toman decisiones observando que los cambios en sus lógicas de trabajo emanan de sus propias necesidades internas.

Los asesores pedagógicos así lo reconocen al enfatizar la necesidad de "Generar un ámbito de indagación crítica de las problemáticas pedagógicas propias de la institución.” (UNER, Facultad de Ingeniería, Área de Asesoría y Orientación Vocacional).

Los asesores pedagógicos, a través de sus Comunicaciones, expresan (en lo referente a la dimensión de investigación) consideraciones al lugar y reconocimiento institucional que connota esta práctica, a su grado de institucionalización, a la naturaleza interdisciplinaria de su producción, a los aspectos estratégicos, a sus objetos de estudio, y a aportes que hacen a la conformación del campo de la Pedagogía y la Didáctica Universitarias.

En cuanto a la respuesta a la demanda institucional, hay equipos que manifiestan que la investigación se constituye en un elemento inicial y central en las acciones orientadas a enfrentar problemas prioritarios demandados por la institución, constituyéndose la actividad en el eje central y un modus operandi de las acciones que encara la asesoría.

Así manifiestan: 
Del trabajo conjunto, de las autoridades y la asesora, surge como temática a trabajar; la necesidad de conocer y comprender los factores y el contexto en que aparece el fenómeno de la deserción de los alumnos ingresantes en la Universidad Nacional del Sur especificamente y las posibles formas de revertirla. Desde la asesoría pedagógica, este fenómeno [la deserción] se constituye en un objeto de investigación. (UNS, Secretaría General Académica, Asesora Pedagógica).

[Son] Proyectos aprobados por el Consejo de Investigaciones de la UNT... (Universidad Nacional de Tucumán - UNT, Facultad de Filosofía y Letras, Instituto Coordinador de Programas de Capacitación - ICPC).

Con respecto a la modalidad estratégica que se desarrolla en torno a la investigación, esta se define, en los casos analizados, a través de las formas de articulación en la conformación de los equipos y de la metodología por ellos adoptada. En los casos analizados el asesor desarrolla una relación de par en un equipo caracterizado por la conjunción de disciplinas singulares, cuyos integrantes son poseedores de distintos saberes que se complementan en el análisis de una práctica de interés común, la formación del estudiante universitario. La multidimensionalidad, nota distintiva de una perspectiva crítica acerca de lo pedagógico (CANDAU, 1995), se hace presente en esta forma de encarar la asesoría para la realización de proyectos de investigación.

Estos testimonios dan cuenta de esta última mirada:

[Se constituye un] Grupo de Investigación para la Enseñanza de la Tecnología en la Universidad y su Articulación con la Escuela Media. [Es un] Equipo multidisciplinario de investigadores en: pedagogía, disciplinas científicas y tecnológicas, orientando en el diseño de Proyectos tecnológicos integradores que involucraron el empleo de materiales piloto, su construcción y utilización. (Facultad de Ingeniería, Universidad de Buenos Aires - FIUBA, Asesoría Pedagógica).

En lo que refiere a las dimensiones metodológicas de los proyectos en sí, que se ponen en juego a la hora de desarrollar el proceso de investigación educativa (SIRVENT, 2006), se reconoce en estos casos analizados el predominio de la lógica cualitativa sobre la cuantitativa, cuya meta principal es la comprensión de los hechos que aborda el asesor pedagógico en su quehacer profesional e institucional.

Así lo manifiesta este testimonio: 
La investigación, desde un punto de vista metodológico, conjuga el enfoque cuantitativo y cualitativo. [...] [El uso del segundo] hizo que tomara visibilidad para las autoridades, uno de los actores principales: el alumno, y, en especial, el sentido que éste le otorga a los fenómenos en cuestión, en oposición a la consideración únicamente numérica del fenómeno como forma de explicar [lo]. (Universidad Nacional del Sur - UNS, Secretaría General Académica - SGA, Asesora Pedagógica).

Esta labor, en la posición que sostiene esta asesora pedagógica, contribuye a la construcción de conocimiento en el campo de la Didáctica Universitaria y la función del asesor no solamente en su participación sistemática sino en el mejoramiento de la propia práctica. En este sentido importó ver el contenido de la investigación, lo que nos llevó a preguntarnos qué investigan los asesores cuando investigan. Se revela así el campo de indagación de la práctica del asesor en su dinámica en relación a las demandas institucionales, conformando un abanico que incluye asuntos específicos como la deserción y las tutorías como recurso institucional para prevenirla, la formación de los docentes, la relación entre saber pedagógico y saber disciplinar en la enseñanza, los medios audiovisuales y otros de carácter más amplio como el modelo pedagógico, las estructuras académicas, la relación educativa, entre otros.

Un último punto de interés en el análisis de esta dimensión se vincula a la percepción que tienen los asesores de la incidencia de sus acciones investigativas en la conformación del campo de la Pedagogía y la Didáctica Universitarias. Si se afirma que la asesoría pedagógica se define a través de su accionar en la institución en función del carácter complejo de su práctica, es desde este cruce de prácticas que surge como actor central en la construcción de la Didáctica y la Pedagogía Universitarias. (LUCARELLI, 2008). Así parecen reconocerlo los equipos analizados, quienes expresan:

[...] el asesor "desde" y "en" su práctica de intervención es un constructor de teoría en temáticas relacionadas con la formación docente, la didáctica universitaria, así como sobre las problemáticas educativas universitarias. (UNS, Secretaría General Académica, Asesora Pedagógica).

Un último testimonio permite ver intencionalidades más allá de las acciones concretas: 
[Son] Proyectos de investigación que revitalizan la Pedagogía Universitaria [...]. La importancia de estas líneas de investigación está en la posibilidad de generar alternativas para la comprensión, gestión y modificación de las prácticas pedagógicas en la universidad, así como producir nuevos dispositivos de formación docente en el marco de la configuración de una Didáctica Universitaria. (UNT, Facultad de Filosofía y Letras, ICPC).

El asesor pedagógico en las universidades nacionales argentinas busca afianzar su lugar institucional a través del entramado de acciones que van desde la intervención directa en los procesos del aula y de la institución en su conjunto, a la investigación que le permite comprender el significado de esos procesos, posibilitando así, desde la acción y la reflexión sistemática, aportar a la construcción del campo de la Pedagogía y la Didáctica Universitarias.

\section{REFERENCIAS}

CANDAU, V. M. (Org.). Rumo a uma nova didática. Petrópolis, RJ: Vozes, 1995.

DA CUNHA, M. I. (Org.). Pedagogia universitária: energias emancipatórias em tempos neoliberais. Arararaquara, SP: Junqueira \& Marin, 2006.

DA CUNHA, M. I.; LUCARELLI, E. La vida de los académicos: prácticas de formación y mejoramiento de la enseñanza. En: CONGRESO MUNDIAL DE EDUCACIÓN COMPARADA, "Nuevos tiempos, nuevas voces. Perspectivas comparadas para la educación", XV., 2013, Buenos Aires. Anales... Buenos Aires, Argentina: Facultad de Ciencias Económicas de la Universidad, 24 al 28 de junio de 2013.

FERNÁNDEZ, L. F. El análisis institucional y el asesoramiento pedagógico universitario. En: LUCARELLI, E.; FINKELSTEIN, E. El asesor pedagógico en la universidad. Entre la formación y la intervención. Buenos Aires: Miño y Dávila, 2012.

HAVELOCK, R. Planning for innovation. Ann Arbor: University of Michigan, 1969.

LANGER, A. El sistema científico y las universidades: revisión de teorías y enfoques en América Latina y Argentina. En: RIQUELME, G. (Ed.). Las universidades frente a las demandas sociales y productivas. Buenos Aires: Miño y Dávila, 2008. Tomo I.

LUCARELLI, E. (Comp.). El asesor pedagógico en la universidad. De la teoría pedagógica a la práctica en la formación. Buenos Aires: Paidós, 2000. 
LUCARELLI, E. Problemáticas en torno a un rol: El asesor pedagógico universitario. En: CIOEI, X., 2008, Barcelona. Anales... Barcelona: Universidad de Barcelona, 2008.

LUCARELLI, E.; FINKELSTEIN, E. El asesor pedagógico en la universidad. Entre la formación y la intervención. Buenos Aires: Miño y Dávila, 2012.

LUCARELLI, E.; FINKELSTEIN, C.; SOLBERG, V. Principales problemas en el campo de acción del asesor pedagógico universitario en Argentina. En: DA CUNHA, M. I.; LUCARELLI, E. A. (Orgs.). Estratégias de qualificação do ensino e o assessoramento pedagógico: reconhecendo experiências em universidades ibero-americanas. Criciúma, SC: UNESC, 2014. (en proceso de edición).

MURILLO ESTEPA, P. El diseño de la función asesora en el nuevo sistema público: posibilidades y problemas. En: MARCELO GARCÍA, C.; LÓPEZ YÁÑEZ, J. (Coords.). Asesoramiento curricular y organizativo en educación. Barcelona: Ariel, 1997.

SÁNCHEZ MORENO, M. El proceso de asesoramiento. En: MARCELO GARCÍA, C.; LÓPEZ YÁÑEZ, J. (Coords.). Asesoramiento curricular y organizativo en educación. Barcelona: Ariel, 1997.

SIRVENT, M. T. El proceso de investigación. Ficha de cátedra. Buenos Aires: OPFYL., FFyL. UBA, 2006.

Texto recebido em 08 de julho de 2015. Texto aprovado em 11 de agosto de 2015. 
